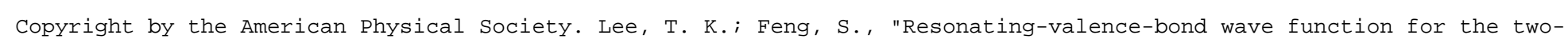
dimensional Heisenberg model on a triangular lattice," Phys. Rev. B 41, 11110 DoI: http://dx.doi.org/10.1103/ PhysRevB. 41.11110

PHYSICAL REVIEW B

\title{
Resonating-valence-bond wave function for the two-dimensional Heisenberg model on a triangular lattice
}

\author{
T. K. Lee \\ Department of Physics, Virginia Polytechnic Institute and State University, Blacksburg, Virginia 24061 \\ Shiping Feng* \\ Texas Center for Superconductivity, University of Houston, Houston, Texas 77204
}

(Received 13 November 1989)

\begin{abstract}
A resonating-valence-bond ( $R$ VB) wave function for the triangular lattice is constructed by following the renormalized Hamiltonian approach (or Gutzwiller approximation) successfully developed for the square lattice. This wave function is then used to calculate the ground-state energy by using the variational Monte Carlo method. Comparison with results of other trial wave functions is discussed. Energy of this paramagnetic RVB state is not as low as other antiferromagnetically ordered wave functions. A flux-phase state similar to the RVB state is also presented. This state with one-quarter flux quantum per plaquette breaks time-reversal symmetry.
\end{abstract}

\section{INTRODUCTION}

Since the discovery that the copper oxygen sheets in the copper oxide superconductors have strong antiferromagnetic spin correlations, ${ }^{1}$ it is believed that the physics of these materials is contained in a two-dimensional, large- $U$, single-band Hubbard model. ${ }^{2}$ For the case of one electron per $\mathrm{Cu}$ site, this Hubbard model is equivalent to the two-dimensional antiferromagnetic Heisenberg model, where the spins interact via the superexchange mechanism. Hence, understanding of the ground-state and low-energy excitations of the Heisenberg model may be essential to establish the superconducting mechanism in these materials.

In one of the earliest papers on this subject Anderson ${ }^{2}$ proposed that the magnetic correlation embedded in the Heisenberg model can be understood in terms of the resonating-valence-bond ( $R V B$ ) state. This state was first proposed by Anderson and Fazekas ${ }^{3}$ to be the ground state of the antiferromagnetic Heisenberg model on a two-dimensional triangular lattice. Subsequently, a substantial amount of work was devoted to understanding the RVB state on a square lattice pertinent to the copper oxide superconductors.

Several superconducting mechanisms ${ }^{4,5}$ based on a RVB state have been proposed. In particular, Laughlin ${ }^{6}$ has recently proposed a new novel superconductor. Kalmeyer and Laughlin ${ }^{7}$ argued that the RVB state for the triangular lattice is "similar" to the fractional quantum Hall state for bosons. Hence, the elementary excitations obey fractional statistics and may form a new superconductor. However, the variational state constructed by Kalmeyer and Laughlin has much higher energy than the long-range-ordered antiferromagnetic state proposed by Huse and Elser. ${ }^{8}$ It raises the question whether a much lower-energy RVB state for the triangular lattice can be constructed.

The situation for the square lattice is a bit more ad- vanced. A systematic way of constructing a RVB state in the fermion representation is proposed by Zhang et al. ${ }^{5}$ Using the Gutzwiller approximation they obtain a paramagnetic RVB state. The variational energy evaluated by the Monte Carlo method ${ }^{9}$ is only $4 \%$ higher than the believed "exact" ground-state energy. Recently we $\mathrm{w}^{10}$ have shown that by modifying the paramagnetic RVB wave function to become a long-range-ordered antiferromagnetic state, energy is gained by more than $3 \%$. More interestingly, doping of holes with $5-7 \%$ can easily restore the stability of the paramagnetic RVB state.

The purpose of this paper is to apply this successful approach for the square lattice to the triangular lattice. In Sec. II we construct the RVB wave function by using the renormalized Hamiltonian approach discussed by Zhang et al. $^{5}$ Three possible solutions of the mean-field theory with different symmetries are presented. In Sec. III, the ground-state energies of these wave functions are calculated numerically by using the variational Monte Carlo methods. ${ }^{9}$ The state of the lowest energy has $d$-wave symmetry; this is similar to the result of the square lattice. We show that the lowest energy obtained is still higher than the result of Huse and Elser ${ }^{8}$ for a longrange-ordered antiferromagnetic state. To conclude this paper, in Sec. IV, we discuss the possible reason for the "shortfall" of our approach and relation of our wave function with that of Kalmeyer and Laughlin. ${ }^{7}$ In the Appendix, we present a flux-phase state $^{11}$ similar to the $d$-wave RVB state. This state is one of the $T$ and $P$ symmetry-broken states discussed by Wen and Zee. ${ }^{12}$

\section{GUTZWILLER MEAN-FIELD THEORY FOR TRIANGULAR LATTICE}

In this section, we use the Gutzwiller approximation ${ }^{13}$ to study the RVB state for the two-dimensional Heisenberg model on a triangular lattice. We follow the procedure developed by Zhang et al. ${ }^{5}$ for a square lattice; 
the trial wave function proposed for the RVB state has the form

$$
\begin{aligned}
& |\psi\rangle=P_{d}\left|\psi_{0}\right\rangle, \\
& \left|\psi_{0}\right\rangle=\prod_{\mathbf{k}}\left(u_{\mathbf{k}}+v_{\mathbf{k}} C_{\mathbf{k} \uparrow}^{\dagger} C_{-\mathbf{k} \downarrow}^{\dagger}\right)|0\rangle,
\end{aligned}
$$

where the operator $P_{d}=\prod_{i}\left(1-n_{i \uparrow} n_{i \downarrow}\right)$ projects out double occupancy on each site, and $u_{\mathrm{k}}$ and $v_{\mathrm{k}}$ are the variational parameters satisfying the normalization condition of $\left|\psi_{0}\right\rangle:\left|u_{k}\right|^{2}+\left|v_{k}\right|^{2}=1$. Following the work of Zhang et al. ${ }^{5}$ the effect of the projection operator on the doubly occupied site is taken into account by a classical statistical weighting factor that multiplies the quantum coherent result calculated with $\left|\psi_{0}\right\rangle$. The spin-spin correlation of the nearest-neighbor sites $i$ and $j$ in the state $|\psi\rangle$ are related to those in the state $\left|\psi_{0}\right\rangle$ by

$$
\left\langle\mathbf{S}_{i} \cdot \mathbf{S}_{j}\right\rangle=g_{s}\left\langle\mathbf{S}_{i} \cdot \mathbf{S}_{j}\right\rangle_{0},
$$

where $\langle A\rangle_{0}$ and $\langle\boldsymbol{A}\rangle$ are the expectation values in the state $\left|\psi_{0}\right\rangle$ and $|\psi\rangle$, respectively. For a half-filled lattice the renormalization factor $g_{s}=4$. Substituting Eqs. (2) into (3), we obtain the ground-state energy $W$ :

$$
\begin{aligned}
W & =\sum_{\langle i j\rangle}\left\langle\mathbf{S}_{i} \cdot \mathbf{S}_{j}\right\rangle \\
& =\frac{1}{N_{s}} \sum_{\mathbf{k}, \mathbf{k}^{\prime}} V_{\mathbf{k}-\mathbf{k}^{\prime}}\left(u_{\mathbf{k}} v_{\mathbf{k}}^{*} v_{\mathbf{k}^{\prime}} u_{\mathbf{k}^{\prime}}^{*}-\left|v_{\mathbf{k}}\right|^{2}\left|u_{\mathbf{k}^{\prime}}\right|^{2}\right),
\end{aligned}
$$

where $N_{s}$ is the total number of sites,

$$
V_{\mathbf{k}}=-3 g_{s} \gamma_{\mathbf{k}} / 4
$$

and

$$
\begin{gathered}
\gamma_{k}=2\left[\cos \left(k_{x}\right)+\cos \left(\frac{k_{x}-\sqrt{3} k_{y}}{2}\right)\right. \\
\left.+\cos \left(\frac{k_{x}+\sqrt{3} k_{y}}{2}\right)\right] .
\end{gathered}
$$

We have used the assumption of even parity, ${ }^{14}$ $u_{\mathrm{k}} v_{\mathrm{k}}^{*}=u_{-\mathrm{k}} v_{-\mathrm{k}}^{*}$ in the derivation of Eq. (4). We shall minimize the total free energy

$$
E=W-\mu N
$$

under the constraints that $\left|u_{\mathbf{k}}\right|^{2}+\left|v_{\mathbf{k}}\right|^{2}=1$ and

$$
N=\left\langle\sum_{\mathbf{k} \sigma} C_{\mathbf{k} \sigma}^{\dagger} C_{\mathbf{k} \sigma}\right\rangle=N_{s}=2 \sum_{\mathbf{k}}\left|v_{\mathbf{k}}\right|^{2} .
$$

To simplify matters further, we shall take

$$
\left|u_{\mathbf{k}}\right|^{2}=\frac{1}{2}\left(1+\xi_{\mathbf{k}} / E_{\mathrm{k}}\right), \quad\left|v_{\mathbf{k}}\right|^{2}=\frac{1}{2}\left(1-\xi_{\mathrm{k}} / E_{\mathbf{k}}\right),
$$

and

$$
u_{\mathrm{k}}^{*} v_{\mathrm{k}}=\Delta_{\mathrm{k}} / 2 E_{\mathrm{k}},
$$

where

$$
E_{k}=\left(\xi_{\mathbf{k}}^{2}+\left|\Delta_{\mathbf{k}}\right|^{2}\right)^{1 / 2} .
$$

The parameters $\Delta_{k}$ and $\xi_{k}$ satisfy the following coupled gap equations:

$$
\begin{aligned}
& \Delta_{\mathbf{k}}=-\frac{1}{N_{s}} \sum_{\mathbf{k}^{\prime}} V_{\mathbf{k}-\mathbf{k}^{\prime}} \frac{\Delta_{\mathbf{k}^{\prime}}}{2 E_{\mathbf{k}^{\prime}}}, \\
& \xi_{\mathbf{k}}=-\mu^{\prime}-\frac{1}{N_{s}} \sum_{\mathbf{k}^{\prime}} V_{\mathbf{k}-\mathbf{k}^{\prime}} \frac{\xi_{\mathbf{k}^{\prime}}}{2 E_{\mathbf{k}^{\prime}}} .
\end{aligned}
$$

The renormalized chemical potential $\mu^{\prime}$ is determined by Eq. (8) or

$$
\sum_{\mathbf{k}} \xi_{\mathbf{k}} / E_{\mathbf{k}}=0
$$

The ground-state energy of the system is given by

$$
W=-\frac{1}{2} \sum_{\mathbf{k}} E_{\mathbf{k}}
$$

The gap equations, (12) and (13), and the constraint (14) can be solved exactly if we assume the quasiparticle energy $E_{\mathrm{k}}$ has the rotational symmetry of the triangular lattice. According to the irreducible representations of the triangular lattice, there are three possible basis functions of even parity: ${ }^{14}$ one $s$-like functions,

$$
s_{k}=\cos k_{x}+\cos \left[\left(k_{x}-k_{y}^{\prime}\right) / 2\right]+\cos \left[\left(k_{x}+k_{y}^{\prime}\right) / 2\right],
$$

and two $d$-like functions,

$$
\begin{aligned}
d_{1 k}= & 2 \cos k_{x}-\cos \left[\left(k_{x}-k_{y}^{\prime}\right) / 2\right] \\
& -\cos \left[\left(k_{x}+k_{y}^{\prime}\right) / 2\right],
\end{aligned}
$$

and

$$
d_{2 k}=\cos \left[\left(k_{x}+k_{y}^{\prime}\right) / 2\right]-\cos \left[\left(k_{x}-k_{y}^{\prime}\right) / 2\right],
$$

where $k_{y}^{\prime}=\sqrt{3} k_{y}$. By assuming that $\Delta_{k}$ and $\xi_{k}$ are linear combinations of these three basis functions, we obtain three possible solutions: (1) extended s-wave symmetry or projected Fermi-liquid state with $\Delta_{\mathbf{k}}=0$ and $\xi_{\mathrm{k}}=-\mu^{\prime}-\xi s_{k}$, where $\xi=\frac{1}{6}$ and $\mu^{\prime}=0.14$ (for this state the energy per site is $W / N_{s}=-0.5$ ); (2) one-component $d$ state with $\xi_{\mathrm{k}}=-\xi d_{1 k}, \Delta_{\mathrm{k}}=\sqrt{3} \xi d_{2 k}$, and quasiparticle energy $E_{\mathbf{k}}=\left(d_{1 k}^{2}+3 d_{2 k}^{2}\right)^{1 / 2}[\xi$ is determined by the equation $4 \xi^{2}=\left(1 / N_{s}\right) \Sigma_{k} E_{\mathrm{k}}$, and it has the value 0.563 ; the energy per site is $W / N_{s}=-0.633$ ]; and (3) $d$-wave state with $\xi_{\mathbf{k}}=-\xi s_{k}$ and $\Delta_{\mathbf{k}}=(\xi / \sqrt{2})\left(d_{1 k}+i \sqrt{3} d_{2 k}\right)$. The quasi-particle energy is of the form

$$
\begin{gathered}
E_{\mathbf{k}}=(3)^{1 / 2} \xi\left[\cos ^{2} k_{x}+\left[\cos \frac{k_{x}-\sqrt{3} k_{y}}{2}\right]^{2}\right. \\
\left.+\left[\cos \frac{k_{x}+\sqrt{3} k_{y}}{2}\right]^{2}\right]^{1 / 2},
\end{gathered}
$$

where $\xi$, determined by the equation $3 \xi^{2}=\left(1 / N_{s}\right) \Sigma_{k} E_{\mathbf{k}}$, has the value 0.693 . This state has the lowest energy per site $W / N_{s}=-0.721$.

It is noticed that the Heisenberg model has local SU(2) or $\mathrm{U}(1)$ gauge invariance, there is many equivalent wave functions with different $\xi_{k}$ and $\Delta_{k}$ but the same $E_{k}$. Since this is similar to the situation that occurred in the square lattice, ${ }^{5}$ we shall not discuss it further.

For the triangular lattice the Gutzwiller approach finds the $d$-wave state to have the lowest energy. This is in fact 
very similar to the $d$-wave solution of the square lattice. We noticed that in both cases, the quasiparticle energy $E_{\mathbf{k}}$ is of the form $E_{\mathbf{k}}^{2} \sim \sum_{i}\left(\cos \mathbf{k} \cdot \mathbf{a}_{i}\right)^{2}$, where $\mathbf{a}_{i}$ include all the nearest-neighbor lattice vectors.

For the Heisenberg model on a square lattice, Affleck and Marston" obtained a "flux phase" state that has same quasiparticle energy as the RVB state. In the Appendix we construct the flux-phase state for the triangular lattice. The state with quasiparticle energy the same as the $d$-wave state of Eq. (16) has one quarter of a flux quantum for each triangular plaquette. It is one of the $P$ and $T$-violating chiral spin states proposed by Wen and Zee. ${ }^{12}$ Noticing the way the gauge field has been chosen, we have assumed two inequivalent lattice sites.

Dividing the lattice into two sublattices is compatible with the symmetry of the Néel state of the square lattice but not the triangular lattice. The Néel state of the triangular lattice $\mathrm{e}^{3,8}$ involves three sublattices. Hence, for the triangular lattice we expect the energy of the $d$-wave RVB state or the flux phase state to be quite different from that of the antiferromagnetic state not as the case of the square lattice, where both states have almost the same energy. ${ }^{10,15}$

The energy just calculated by using the Gutzwiller approximation is not accurate enough to compare with other trial wave functions. To calculate the energy of the RVB state reliably we shall use the variational Monte Carlo method.

\section{VARIATIONAL MONTE CARLO RESULT}

The expectation value of $\left\langle\mathbf{S}_{i} \cdot \mathbf{S}_{j}\right\rangle$ in the state $|\psi\rangle$ of Eqs. (1) and (2) can be evaluated numerically by using the Monte Carlo method. Details of this method can be found in Ref. 9.

In our calculation, the total number of particle is fixed, and the wave function $\psi$ of Eq. (11) is rewritten in the form

$$
|\psi\rangle=P_{d}\left(\sum_{i, j} a_{i j} C_{i \uparrow}^{\dagger} C_{j \downarrow}^{\dagger}\right)^{N_{s} / 2}|0\rangle
$$

where $i, j$ are all possible pairs of sites. The matrix element $a_{i j}=a\left(R_{i}-R_{J}\right)$ is a Fourier transform of

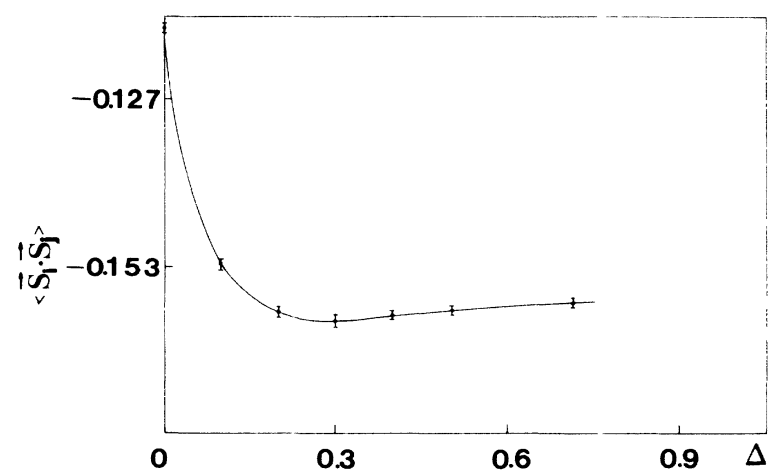

FIG. 1. $\left\langle\mathbf{S}_{i} \cdot \mathbf{S}_{J}\right\rangle$ plotted as a function of the variational parameter $\Delta$ for the $d$-wave RVB state with 90 sites.
TABLE I. A comparison of the ground-state energy for the Heisenberg model on the two-dimensional triangular lattice.

\begin{tabular}{lll}
\hline \multicolumn{1}{c}{ Author(s) } & $-\left\langle\mathbf{S}_{i} \cdot \mathbf{S}_{j}\right\rangle$ & \multicolumn{1}{c}{ Method } \\
\hline Kalmeyer and Laughlin $^{\mathrm{a}}$ & $0.47(1)$ & Variational RVB \\
Huse and Elser $^{\mathrm{b}}$ & $0.51(1)$ & Variational AFM \\
Nishimori and Nakanishi $^{\mathrm{c}}$ & $0.55(1)$ & Finite lattice \\
Present work & $0.484(2)$ & Variational RVB \\
\hline \hline
\end{tabular}

${ }^{\mathrm{a}}$ Reference 7.

${ }^{b}$ Reference 8.

${ }^{\mathrm{c}}$ Reference 16 .

$$
a(\mathbf{k})=v_{\mathrm{k}} / u_{\mathrm{k}}=\Delta_{\mathrm{k}} /\left(E_{\mathrm{k}}+\xi_{\mathrm{k}}\right) .
$$

Each of the three solutions discussed in Sec. II is used to calculate $\left\langle\mathbf{S}_{i} \cdot \mathbf{S}_{j}\right\rangle$. The extended $s$-wave state has $\left\langle\mathbf{S}_{i} \cdot \mathbf{S}_{j}\right\rangle=-0.346(2)$. The one-component $d$ state, where $\xi_{\mathrm{k}}=-d_{1 k}$ and $\Delta_{\mathrm{k}}=\Delta d_{2 k}$, has its energy minimum $\left\langle\mathbf{S}_{i} \cdot \mathbf{S}_{j}\right\rangle=-0.472(2)$ at $\Delta=1.5$.

The minimum energy is achieved by the $d$-wave state. Choosing $\xi_{\mathrm{k}}=-s_{k}$ and $\Delta_{\mathrm{k}}=\Delta\left(d_{1 k}+i \sqrt{3} d_{2 k}\right)$, we have $\left\langle\mathbf{S}_{i} \cdot \mathbf{S}_{j}\right\rangle=-0.484(1)$ for $\Delta=0.3$. In Fig. $1,\left\langle\mathbf{S}_{i} \cdot \mathbf{S}_{j}\right\rangle$ is plotted as a function of $\Delta$ for the $d$-wave state with $N_{s}=90$ sites. Size dependence of our result is also studied and found not significant.

In Table I the values of $\left\langle\mathbf{S}_{i} \cdot \mathbf{S}_{j}\right\rangle$ calculated using different methods are compared. The antiferromagnetic (AFM) state proposed by Huse and Elser ${ }^{8}$ has considerably lower energy than the $d$-wave RVB state. Using the wave function of fractional quantized Hall effect for this problem, Kalmeyer and Laughlin obtained $\left\langle\mathbf{S}_{i} \cdot \mathbf{S}_{j}\right\rangle$ $=-0.47(1)$, almost the same as the result of $d$-wave state.

\section{CONCLUSION}

The renormalized Hamiltonian approach (or Gutzwiller approximation) developed for the Heisenberg model on a square lattice is extended to the triangular lattice to construct a RVB wave function. Not surprisingly, RVB wave functions obtained for these two lattices are quite similar. However, for the square lattice the energy of the RVB state is only $4 \%$ higher than the long-range-ordered AFM state, while it is probably more than $10 \%$ higher for the triangular lattice.

More interestingly, the excitation spectra of the RVB state for the square lattice is gapless, similar to AFM state. In this case a slight modification of the RVB state to incorporate long-range AFM order easily reduces its energy to within $1 \%$ of the exact ground state, but in the triangular lattice, the excitation spectra of the RVB state has a large gap. We found it to be quite stable against AFM ordering. In other words the RVB state obtained in the renormalized Hamiltonian approach seems incompatible with the AFM state. A much better trial wave function may be obtained in the flux-phase approach.

In the Appendix, a flux-phase state with one quarter of a flux quantum per plaquette is shown to have the same quasiparticle energy as the RVB state (see also Fig. 2). Just as for the square lattice, we believe that this one- 


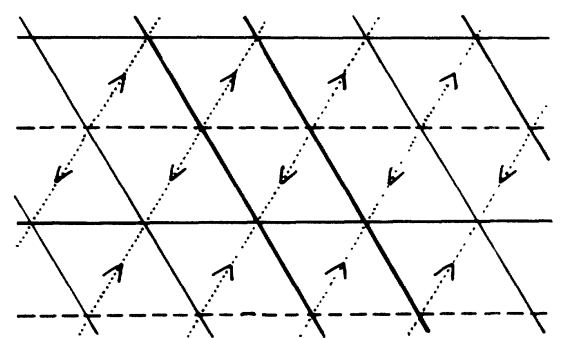

FIG. 2. Choices of the phase factor for the quantity $Q_{i j}$ defined in the Appendix. The solid line has phase zero, the dashed line has phase -1 , and the dotted line has phase $-i$ in the direction of the arrow.

quarter-flux-phase state is identical with the RVB state. However, this state divides the triangular lattice into two sublattices. The AFM state divides the lattice into three inequivalent sublattices. Hence a generalized flux-phase state taking into account this AFM property probably will have much lower energy than what we have obtained now.

It is also interesting to notice that the state proposed by Kalmeyer and Laughlin $^{7}$ (KL) also has two sublattices. In their state the boson gained a phase of $2 \pi$ by moving around two adjacent plaquettes, and in our case a phase of $\pi$ is obtained for the fermions. Also the values of the variational energies are so close between these two states that it suggests that $\mathrm{KL}$ state and $d$-wave RVB state may be of the same class.

\section{ACKNOWLEDGMENTS}

The authors wish to thank Professor C. S. Ting and Professor F. C. Zhang for many useful discussions. T.K.L. would also like to thank the Texas Center for Superconductivity at the University of Houston, where most of the work was done. This work was partially supported by the Texas Center for Superconductivity at the University of Houston under prime Grant No. MDA 972-88-G-0002 to the University of Houston from the Defense Advanced Research Projects Agency.

\section{APPENDIX: THE FLUX-PHASE STATE}

Affleck and Marston ${ }^{11}$ proposed the flux-phase state as their trial wave function for Heisenberg model on a square lattice. The wave function is constructed by choosing the auxiliary field $Q_{i j}$ to minimize the energy

$$
H_{0}=\sum_{\langle i j\rangle}\left(2\left|Q_{i j}\right|^{2}+\sum_{\sigma}\left(Q_{i j} C_{j \sigma}^{\dagger} C_{i \sigma}+\text { H.c. }\right)\right],
$$

and $Q_{i j}=-\frac{1}{2} \sum_{\sigma}\left\langle C_{l \sigma}^{\dagger} C_{j \sigma}\right\rangle$ is the effective hopping amplitude between nearest-neighbor sites $i$ and $j$. For the square lattice, one possible solution is to choose the phase of $Q_{i j}$ to give half a flux quantum per square plaquette. The spectrum obtained is identical to the $d$-wave RVB state.

A flux-phase state with a similar energy spectrum to the $d$-wave state of Eq. (16) can be obtained by having the magnitude of $\left|Q_{i}\right|=Q$ the same for all the bonds and the phase of $Q_{i j}$ as shown in Fig. 2. In Fig. 2, the solid line has phase zero, the dashed line has phase -1 , and the dotted line has phase $-i$ in the direction of the arrow. The electrons obtain a net phase of $-i$ by moving around each triangular plaquett. Hence in this state the electrons are moving under a magnetic field with one quarter of flux quantum per plaquette.

With these choices of $Q_{i j}$ Eq. (A1) can be written in the form

$$
\begin{aligned}
H_{0}=N_{s} 6 Q^{2}+\sum_{k \sigma}[ & C_{\mathrm{k} \sigma}^{\dagger} C_{\mathbf{k} \sigma} 2 Q \cos \mathbf{k} \cdot \mathbf{a}_{3} \\
+ & C_{\mathbf{k}+\mathrm{Q}, \sigma}^{\dagger} C_{\mathbf{k} \sigma} \\
& \left.\times Q\left(\cos \mathbf{k} \cdot \mathbf{a}_{1}-i \cos \mathbf{k} \cdot \mathbf{a}_{2}\right)+\text { H.c. }\right]
\end{aligned}
$$

where the nearest-neighbor lattice vectors are $\mathbf{a}_{1}=(1,0)$, $\mathbf{a}_{2}=(1 / 2, \sqrt{3} / 2)$, and $\mathbf{a}_{3}=(-1 / 2, \sqrt{3} / 2)$. The vector $\mathbf{Q}=(0,2 \pi / \sqrt{3})$. Equation (A2) can be easily diagonalized, and its eigenvalue is the same as $E_{\mathrm{k}}$ of Eq. (16).

From Fig. 2 we can see that the choice of phase has divided the lattice into two sublattices. This is quite different from the three sublattices associated with the Néel state. ${ }^{3}$ Hence, we suspect this flux-phase state may not have the optimum antiferromagnetic correlation.
*Permanent address: Department of Physics, Beijing Normal University, Beijing, China.

${ }^{1}$ D. Vaknin et al., Phys. Rev. Lett. 58, 2802 (1987); A. Aharony et al., ibid. 60, 1330 (1988).

${ }^{2}$ P. W. Anderson, Science 235, 1196 (1987).

${ }^{3}$ P. W. Anderson, Mater. Res. Bull. 8, 153 (1973); P. Fazekas and P. W. Anderson, Philos. Mag. 30, 423 (1974).

${ }^{4}$ P. W. Anderson, G. Baskaran, Z. Sou, and T. Hsu, Phys. Rev. Lett. 58, 2790 (1987); T. M. Rice, Z. Phys. B 67, 141 (1987).

${ }^{5}$ F. C. Zhang, C. Gros, T. M. Rice, and H. Shiba, Superconductor Sci. Technol. 1, 36 (1988).

${ }^{6}$ R. B. Laughlin, Phys. Rev. Lett. 60, 2677 (1988); Science 242, 526 (1988).

${ }^{7}$ V. Kalmeyer and R. B. Laughlin, Phys. Rev. Lett. 59, 2095
(1987), and references therein.

${ }^{8}$ D. A. Huse and V. Elser, Phys. Rev. Lett. 60, 2531 (1988).

${ }^{9}$ C. Gros, Phys. Rev. B 38, 931 (1988); H. Yokoyama and H. Shiba, J. Phys. Soc. Jpn. 57, 2482 (1988).

${ }^{10}$ T. K. Lee and Shiping Feng, Phys. Rev. B 38, 11809 (1988).

${ }^{11}$ I. Affleck and J. B. Marston, Phys. Rev. B 37, 3774 (1988); 39, 11538 (1989).

${ }^{12}$ X. G. Wen and A. Zee, Phys. Rev. Lett. 62, 2873 (1989).

${ }^{13}$ M. C. Gutzwiller, Phys. Rev. Lett. 10, 159 (1963).

${ }^{14}$ It can be shown that there is no odd-parity solution.

${ }^{15}$ S. Liang, B. Doucot, and P. W. Anderson, Phys. Rev. Lett. 61, 365 (1988).

${ }^{16}$ H. Nishimari and H. Nakanishi, J. Phys. Soc. Jpn. 57, 626 (1988). 